\title{
Ideas de los alumnos sobre los cambios de estado del agua y su evolución con un proceso de formación
}

\author{
José Manuel Carmo \\ Escola Superior de Educação, Universidade do Algarve, Portugal
}

[Recibido el 23 de agosto de 2019, aceptado el 14 de enero de 2021]

En el contexto de un taller de formación, enfocado en la enseñanza de los cambios de estado del agua, enmarcado por una metodología por cambio conceptual, en que se procura dar respuesta a las ideas de los alumnos como hipótesis a investigar y asumiendo la necesidad de introducir la teoría corpuscular de la materia como teoría explicativa de los fenómenos involucrados, los docentes participantes aplican con sus alumnos las actividades entrenadas. El cuestionario inicial para la identificación de los conceptos por los alumnos, se ha aplicado también como post test de 5 a 6 meses después de la realización de las actividades. Los resultados en el pre test se compararon con otras dos investigaciones, utilizando el mismo cuestionario. Los resultados en el post test, revelan un cambio muy destacado en las concepciones de los alumnos, sobrepasando otros de edad superior.

Palabras clave: enseñanza experimental; cambio conceptual; cambios de estado del agua; ideas de los alumnos.

\section{Children's conceptions and perceptions of changes in the state of water before and after instruction}

The article reports on an educational workshop on changes in the state of water. The workshop used a conceptual change teaching methodology designed to treat the pupils' ideas as objects of inquiry worthy of consideration, and corpuscular theory of matter to explain the phenomena involved. To test the effectiveness of the learning activities, the pupils were surveyed using the same pre- and post-test questionnaire. The post-test was applied 5-6 months after the workshop. The results in the pre-test were compared with the findings from two other studies using the same questionnaire. The results of the post-test revealed a very significant improvement in the participants' understanding of the topic, well above expectations for their age group.

Keywords: experimental education; conceptual change; changes in the state of water; pupils' perceptions.

Para citar el artículo. Carmo, J.M. (2021). Ideas de los alumnos sobre los cambios de estado del agua y su evolución con un proceso de formación. Ápice. Revista de Educación Científica, 5(1), 87-99. DOI: https://doi.org/10.17979/arec.2021.5.1.5715

Contacto.jmbcarmo@gmail.com 


\section{Introducción}

El ciclo del agua en la naturaleza se estudia en Portugal en 3ro y en 5to grados de escolaridad, como en una gran diversidad de países. Su estudio exige que se haga referencia a los diferentes estados de la materia y a las transformaciones correspondientes a los cambios de estado, en que sólido, líquido y gaseoso se definen formalmente por las propiedades macroscópicas que les son características, como forma, volumen y "peso". También el concepto de disolución es abordado en estos grados, junto al ciclo da agua, como parte de la comprensión del agua en la naturaleza.

Diferentes investigadores han puesto en evidencia las dificultades en la comprensión del ciclo del agua. Forbes, Zangori y Schwarz (2015) encuentran que los dibujos representativos del ciclo del agua de alumnos de 3er grado presentan de modo destacado referencias a los conceptos estructurales (1) formas del agua y (2) agua en movimiento, mientras las referencias a (3) interacciones agua/geosfera son muy pocas, sin embargo, en todos se verifica un aumento significativo tras un proceso de formación. Los autores encuentran una relación entre el desarrollo de los conceptos estructurales y los componentes de proceso que expresan relaciones temporales entre componentes, movimientos y transformaciones, como evaporación, condensación y precipitación y con las afirmaciones que explicitan como los elementos representados se relacionan con los fenómenos físicos, lo que, en el entender de los autores, expresa una lectura más abstracta de su dibujo. Por el análisis de las representaciones de los alumnos, Márquez y Bach (2007) establecen seis categorías de comprensión del ciclo del agua con base en los componentes espaciales y los componentes dinámicos, el funcionamiento del sistema y la utilización del modelo en otras situaciones. Tras un proceso de formación con estudiantes de 12 a 14 años (1ero de ESO), se encuentra una evolución a categorías más elaboradas integrando circulación atmosférica, superficial y subterránea. Los resultados muestran el aumento concomitante de la percepción de la presencia de los componentes espaciales nubes $(100 \%)$ y vapor $(55 \%)$ y de los componentes dinámicos evaporación (83\%) y, en contrapunto, un aumento muy moderado de la condensación (14\%), aparentemente sustituida por precipitación como proceso alternativo. González (2015), replicando la metodología utilizada por Márquez y Bach (2007) con tan solo 5 alumnos de 3er grado, desvela como los modelos explicativos del ciclo del agua y su evolución en un proceso de formación están relacionados con las deficiencias en la comprensión sobre los cambios de estado del agua, en particular sobre la naturaleza del agua después que se evapora y sobre la condensación. Los datos de Castelltor (2015) refuerzan la idea que la introducción de actividades para la comprensión de los diferentes estados y las transformaciones, en concreto, la evaporación, la condensación, y el papel de la energía del sol, en relación con sus vivencias, contribuyen a representaciones más elaboradas de sus modelos del ciclo del agua y a una mayor capacidad para argumentar sobre la problemática ambiental del agua. En el sistema de progresión de aprendizaje de Gunckel, Covitt, Salinas y Anderson (2013), para una adecuada cultura científica en relación al ciclo del agua, se exigen niveles de comprensión integrando una visión corpuscular de la materia y el concepto de fuerza motora de las transformaciones, así como relacionar los elementos representados con el mundo físico, y la escala microscópica con la macroscópica. Gaviria (2013) describe que tras un proceso de formación con alumnos de 5to grado (10-11 años) en que se asocian los estados de la materia y sus cambios a una teoría explicativa basada en la teoría corpuscular de la materia, los alumnos, al ser solicitados a explicar y dibujar cómo son las partículas y las fuerzas intermoleculares de cada uno de los estados de agregación, el 57\% muestran un progreso en los niveles de representación, evidenciándose el uso de un nivel microscópico fundamentado en la descripción de la organización de las partículas, su movimiento e interacciones. El 23\% más logran representar las partículas y caracterizar los estados de la materia solo por su 
alejamiento relativo. En relación a los cambios de estado, aunque describan los cambios, no lo hacen con la teoría corpuscular de la materia, sino al nivel macroscópico. La casi totalidad (83\%) reconoce la evaporación como cambio del estado líquido al estado gaseoso, en que las partículas se evaporan de la superficie del líquido por acción del calor (del sol) y del viento. Además, logran distinguirla de la ebullición. Aunque reconozcan el retorno del agua, se verifica en los dibujos presentados por la autora que la condensación suele identificarse con precipitación desde las nubes, compuestas por vapor de agua. En un proceso de enseñanza enfocado en las ideas principales que conforman el modelo cinético-corpuscular y su utilización como modelo explicativo en fenómenos cotidianos o naturales, y en que los estudiantes tienen oportunidades para imaginar interpretaciones de los fenómenos a nivel "micro", los dibujos esquemáticos o diagramas explicativos de situaciones distintas a las estudiadas, muestran que cerca del $96 \%$ de los estudiantes de 7 mo grado (10 de ESO), hizo dibujos en que identifican las partículas y, de estos, el $83 \%$ se refieren al movimiento de las partículas. En las explicaciones de sus dibujos un $93 \%$ utilizaron las ideas clave de la teoría corpuscular, de los cuales el 57\% aplicó todas las ideas claves. En preguntas de aplicación sobre situaciones muy distintas a las estudiadas, como transpiración, cambios de estado, naturaleza de los gases o la dilatación, un $46 \%$ aplicó todas las ideas clave en su interpretación (Nebot y Márquez, 2017). Hadenfeldt, Neumann, Sascha, Liu e Ilka (2016) evidencian que la comprensión adecuada de la naturaleza de la materia y sus transformaciones depende de la adquisición de los conocimientos que les permitan explicar los fenómenos en términos de un modelo corpuscular. El grado de desarrollo de las conceptualizaciones sobre estructura y composición, propiedades físicas y cambio, reacciones químicas, y conservación de la materia son las variables de este proceso de progresión, por lo que, el año de escolaridad de su introducción determina diferencias significativas.

El recorrido por las contribuciones de estas investigaciones permite afirmar la necesidad de una mejor comprensión de la condensación y, para eso, la necesidad de introducir la teoría corpuscular de la materia para lograr un aprendizaje adecuado del ciclo del agua. Como parece sugerir el trabajo de Benarroch (2000), la apropiación por los estudiantes de un nivel explicativo en términos de la teoría corpuscular sobre la naturaleza de la materia y sus cambios, se hace necesaria para un acercamiento progresivo y sostenido hacia la concepción académica cuando se enfrentan a nuevas situaciones y a nuevas propuestas curriculares. En resultado de un proceso de formación involucrando a los alumnos de 5to grado en un "mix" de actividades prácticas, demostraciones, videos, exposiciones, debate en clase y escrita. Las entrevistas efectuadas algunas semanas después, revelan que el $67 \%$ usan la teoría corpuscular en la explicación de los fenómenos abordados (Johnson y Papageorgiou, 2010). Las dificultades en comprender la naturaleza de la materia, la sustancia y los cambios de estado, están asociados a un modelo de representación microscópica de la materia insuficientemente estructurado por las ideas clave de la teoría corpuscular de la materia (Cañada, Melo y Álvarez, 2013; Dávila, Cañada y Sánchez 2017).

Confrontados con el fenómeno de la evaporación, de un 30 a 40\% de los alumnos, considera que el agua fue para el aire en partículas muy pequeñas, mientras que de un 60 a $70 \%$ admiten que se transforma en oxígeno e hidrógeno, simplemente deja de existir o es absorbida por el plato (Osborne y Cosgrove, 1983; Zamorano, Dell'Oro y Silva, 1994). Frente a la ebullición, solo un $15 \%$ consideran las burbujas formadas por "vapor", mientras $85 \%$ las consideran formadas por aire, calor u oxígeno e hidrógeno, en porcentajes semejantes (Osborne y Cosgrove, 1983). En este mismo estudio los autores encuentran que solo un porcentaje muy pequeño admite la condensación en la superficie externa de un vaso con hielo tapado, la gran mayoría considerando que el agua del hielo que se derrite y pasa a través del vidrio para fuera, que el frio atraviesa el vidrio y se transforma en agua o que el frio hace que el oxígeno y el hidrógeno del aire se transformen en 
agua. Los resultados obtenidos por Zamorano et al. (1994) coinciden con los obtenidos por Osborne y Cosgrove, (1983). Los resultados del proyecto SPACE (Russel y Watt, 1990) a la respuesta a la pregunta abierta ¿para dónde va el agua?, el $28 \%$ de los alumnos consideran que va para el aire, sin embargo, las referencias dominantes son "nubes" "sol" y "cielo", que adicionadas a que escurre al suelo suman el $46 \%$, por lo que un porcentaje en torno al $75 \%$ admite la conservación del agua en la misma dimensión de los obtenidos por Osborne y Cosgrove, (1983) y Zamorano et al. (1994). La no conservación explícita se evidencia por el hecho que $23 \%$ de los alumnos de más edad continúa a considera que el agua "simplemente se seca y deja de existir". En el estudio de Russel y Watt (1990), tanto los alumnos de 8 a 9 años, como los de 10 a 11, se dividen entre considerar que no es posible recuperar el agua evaporada o que se recupera como lluvia. La condensación no es referida o lo es de modo residual.

No obstante, la tendencia a postergar la introducción de la "teoría corpuscular de la materia" a los 7 mo o 8vo grados, por la supuesta dificultad en su comprensión conceptual por los alumnos más jóvenes, Johnson (1998) sugiere que la presentación de modo independiente de los estados de la materia, de los cambios de estado y de otros fenómenos, como la disolución, evitando la "teoría corpuscular de la materia" como teoría globalizante, es un factor de confusión, promotora de dificultades de aprendizaje, que contribuye a los problemas que posteriormente, se identifican de modo generalizado. En consecuencia, propone su introducción precoz, con el sentido de garantizar una secuencia de comprensiones sucesivas y graduales en una progresión en dirección a la concepción científica. Sin conocer los aspectos claves de la teoría de las partículas como teoría que explica globalmente los hechos, los alumnos y los adultos, tienden a elaborar sus propias teorías explicativas desde su experiencia sensorial, así como a desenvolver teorías alternativas robustas basadas en modelos realistas o macroscópicos.

Las concepciones de los niños evolucionan a partir de los datos que van obteniendo y la enseñanza deberá dar oportunidad a que los alumnos elaboren sobre sus concepciones de modo que gradualmente, las desarrollen integrando los nuevos elementos como ideas particulares sueltas con sentido, "knowledge in pieces", en la designación de Varelas (2006) o "'piecemeal knowledge" en la designación de Venville (2004), originando una comprensión emergente, como una "teoría de transición" (Venville, 2004) en dirección a la construcción de una deseable explicación científica. Es en este sentido que estos autores, apuntan que entendamos en este nivel etario, el modelo de cambio conceptual (Cachapuz, Praia y Jorge, 2000 y 2002), como un proceso de progresión gradual (Johnson, 1998; Johnson y Tymms, 2011) a través de estadios correspondientes a la integración de sucesivos componentes de la comprensión, dependiendo de factores, como el desarrollo cognitivo, la información, el conflicto cognitivo, personal y social.

Piedras y Mota (2011), desde la literatura enuncian un conjunto de categorías de ideas frecuentes en los alumnos que dificultan la adquisición de las concepciones de los científicos acerca de los cambios de estado:

1. No conciben la conservación de la masa ni de la sustancia, por lo que la materia deja de existir cuando desaparece de la vista;

2. Las diversas propiedades de la materia tienen existencia independiente. La materia puede 'desaparecer', mientras que sus propiedades, como la dulzura, pueden continuar existiendo;

3. La existencia de materia sin peso puede ser aceptada, así como los cambios de estado y la disolución están asociados a cambios de peso. Los gases son más leves y los sólidos pesados.

4. Conciben que las partículas también cambian de tamaño; 
5. Adecuan los términos científicos a sus percepciones;

6. Sustancializan el calor;

7. No perciben la reversibilidad en los cambios de estado y soluciones.

Las ideas previas de los niños sobre la naturaleza de la materia y las dificultades en entender las ideas científicas resultan de la dificultad en comprender los siguientes cuatro aspectos (Stavy, 1990; Kind. 2004):

- La materia está hecha de partículas discretas; cada substancia se caracteriza por su molécula.

- Existen "fuerzas" o "enlaces" entre las partículas.

- Las partículas están en constante movimiento al azar, dependiente de la energía del sistema.

- El espacio entre las partículas está vacío.

En este estudio se da cuenta de los resultados obtenidos en un proceso de formación, promovido por el "Centro Ciência Viva de Tavira" para docentes de 3ro y 4to grados de escuelas del municipio con el propósito de promover la enseñanza de tipo práctico y experimental de las ciencias bajo un modelo de cambio conceptual, orientado para los conceptos de evaporación y condensación por su importancia en la conceptualización del ciclo del agua en la Naturaleza. Los docentes involucrados en la formación aplican las actividades integradas en su modelo habitual de enseñanza del ciclo del agua como un complemento.

En la fase inicial de exploración y desconstrucción se procura:

1- Identificar las ideas de los alumnos;

2- Crear oportunidades para que los alumnos exploren sus ideas y verifiquen su solidez en la explicación de los fenómenos, acontecimientos y previsiones;

- Expresan sus conocimientos sobre el asunto o fenómeno;

- Constatan que las previsiones y explicaciones difieren de alumno a alumno;

- Confrontan sus expectativas con las observaciones.

Con esta orientación metodológica, la secuencia de actividades propuesta se inicia por un cuestionario que tiene como objetivo identificar las concepciones previas de los alumnos respecto a los conceptos involucrados en eventos comunes a sus vivencias. Las preguntas del cuestionario reproducen las del trabajo de Osborne y Cosgrove, (1983) por considerarse que representan ideas muy comunes en los alumnos y por suscitaren actividades sencillas, susceptibles de proporcionar indagaciones en clase.

Las actividades subsecuentes se proponen para contribuir a:

3- Ofrecer estímulos para que desarrollen y modifiquen sus ideas cuando necesario;

4- Apoyar la reconstrucción de sus ideas y puntos de vista.

Las actividades propuestas fueran diseñadas como investigaciones para responder a las ideas alternativas de los alumnos, como hipótesis a refutar y la presentación de la teoría del profesor, con capacidad explicativa comprensiva. El subsecuente diálogo en torno de la teoría de las partículas y su aplicación en la interpretación de nuevas situaciones proporcionan oportunidades para que los alumnos reflexionen en el proceso de su pensamiento y desarrollen representaciones en dirección a la concepción científicamente deseada.

\section{Objetivos}

Este trabajo comunica los resultados obtenidos por la aplicación del cuestionario a los alumnos de los profesores en formación al inicio y después de la aplicación de la secuencia de actividades entrenada en la formación y discute la evolución de los resultados. 
Además, se comparan los resultados obtenidos en la respuesta inicial al cuestionario con los resultados de las investigaciones de Osborne y Cosgrove (1983) con estudiantes de 13 años y de Zamorano et al. (1994) con estudiantes de 7 mo grado.

\section{Metodología}

Un taller de formación ubicado en el "Centro Ciência Viva de Tavira", involucra los alumnos de 7 docentes de 3 ro y 4 to grados ( 8 a 9 y 9 a 10 años, respectivamente) en actividades orientadas para la introducción de actividades prácticas de naturaleza experimental en un modelo metodológico de cambio conceptual enfocado en los conceptos de evaporación y condensación.

Los alumnos involucrados han contestado al cuestionario aplicado por sus profesores pre test $(\mathrm{N}=136)$ - en Octubre/Noviembre, como introducción a las actividades proporcionadas, y vuelven a contestar, como post test $(\mathrm{N}=135)$, cerca cinco meses después de la realización de las actividades, en Abril/Mayo aplicado por el investigador.

Las preguntas del cuestionario (anexo, columna izquierda) fueran recogidas directamente del trabajo referido de Osborne y Cosgrove (1983), coincidiendo con las seleccionadas en el estudio de Zamorano et al. (1994), permitiendo una comparación de resultados. En la pregunta 3 se ha introducido una opción de respuesta más -El frasco continúa seco por fuera- buscando identificar la percepción de la ocurrencia del fenómeno.

La primera actividad propuesta en la secuencia de actividades consiste en una auditoría sobre "¿Qué habrá pasado con el agua?" que se ha dejado en un plato desde el día anterior, continuada por debate entre los alumnos. La hoja de trabajo también pide que los alumnos valoren la hipótesis que el agua esté en el aire en nuestro entorno. Los alumnos escriben sus ideas y las comparten con la clase. Mientras se desarrolla el trabajo, se constatará el olor de la evaporación de alcohol, perfume y gasolina, colocados en platos en puntos distintos de la clase; se debate sobre donde ha ido lo que se evaporó, y además sobre cómo se explica que sentimos su olor en el aire. Se hace, entonces el primer abordaje a la teoría corpuscular de la materia, al conceptualizar la idea que, al evaporarse un producto, las partículas, o moléculas que lo constituyen se liberan y quedan aisladas, mezcladas con el aire que está en nuestro entorno, con el apoyo de imágenes y un video explicativo. Los alumnos investigan los factores que influyen la evaporación, debatiendo su acción en el marco de la "teoría corpuscular". En continuidad, los alumnos realizan una actividad semejante al propuesto en la pregunta 3 . Hecha la observación que el vaso "queda sudado por afuera" se solicita la invención de un modo de comprobar cada una de las ideas alternativas, como hipótesis. Tres nuevos experimentos permiten refutar en clase las ideas alternativas.

Otras actividades estudian las condiciones que contribuyen a la condensación en contextos cotidianos, como el empañar de cristales, la formación de rocío, vaho o neblina. Construyen modelos de salinas y ciclo del agua, articulando los conceptos y los explicando con base en la teoría. Se les propone inventar un modelo para obtención de agua limpia a partir de agua sucia y salada.

\section{Resultados}

Los gráficos en la columna izquierda del anexo representan el número de alumnos que eligen en el pre test, cada una de las opciones, en cada una de las siete clases involucradas en el estudio. La diferente dimensión de las clases solo admite la constatación de la variabilidad entre clases y la dispersión dentro de cada una de las clases. 
En las tablas 1, 2 y 3 se presentan en porcentajelos resultados, obtenidos en este estudio en la aplicación del pre y post test (columnas 3 ) en paralelo con los resultados de los dos estudios anteriores referidos. En la 1ra pregunta (tabla 1), la gran mayoría (88\%) de los alumnos en este estudio, considera que las burbujas en el agua hirviendo están formadas por vapor, de modo muy diferentes a los resultados obtenidos en los estudios referidos con alumnos de 13 años. No obstante, las diferencias puntuales en la opción "calor" en un caso, y la opción "vapor" en otro, los alumnos de más edad distribuyen sus elecciones de modo equilibrado por todas las opciones.

Tabla 1 - Resultados en porcentaje obtenidos a cada una de las opciones de respuesta sobre a la pregunta “¿De qué están formadas las burbujas cuando el agua hierve?”

\begin{tabular}{|l|c|c|c|c|}
\hline Opciones de respuesta & $\begin{array}{c}1 \\
13 \text { años }\end{array}$ & $\begin{array}{c}2 \\
13 \text { años }\end{array}$ & \multicolumn{2}{|c|}{$\begin{array}{c}3 \\
\text { Grados 3 y 4 } \\
\text { Pre - Post }\end{array}$} \\
\hline Aire & 25 & 37 & 0 & 4 \\
\hline Vapor & 8 & 27 & 88 & 87 \\
\hline Calor & 28 & 11 & 7 & 7 \\
\hline Oxigeno o hidrogeno & 39 & 25 & 5 & 2 \\
\hline
\end{tabular}

1) Resultados de Osborne y Cosgrove, 1983; 2) Resultados de Zamorano et al., 1994; 3) Resultados en este estudio.

En la pregunta 2 (tabla 2), cerca de la mitad de los alumnos ya poseen una concepción de evaporación al considerar que el agua" va para el aire en partículas muy pequeñas", no se diferenciando de los alumnos de más edad en los estudios anteriores referidos. Sin embargo, en este estudio, un porcentaje de $35 \%$ cree que el agua "simplemente se seca y deja de existir" o "es absorbida por el plato" comparable con los resultados en el estudio de Russell y Watt (1990). Pero, el porcentaje de alumnos que considera que el agua que seca deja de existir no difiere de los de más edad en los estudios anteriores referidos de Osborne y Cosgrove, (1983) y Zamorano et al. (1994). Los alumnos de más edad, por consecuencia con más escolaridad, revelan una mayor adhesión a la idea que el agua "se transforma en oxígeno e hidrógeno".

Tabla 2 - Resultados en porcentaje obtenidos a cada una de las opciones de respuesta a la pregunta “¿Qué le ocurre al agua que no escurrió y quedo en el plato?"

\begin{tabular}{|l|c|c|c|c|}
\hline Opciones de respuesta & $\begin{array}{c}1 \\
13 \text { años }\end{array}$ & $\begin{array}{c}2 \\
13 \text { años }\end{array}$ & \multicolumn{2}{|c|}{$\begin{array}{c}3 \\
\text { Grados 3 y 4 } \\
\text { Pre - Post }\end{array}$} \\
\hline Es absorbida por el plato & 3 & 0 & 15 & 1 \\
\hline Simplemente se seca y deja de existir & 23 & 23 & 20 & 1 \\
\hline Se transforma en oxígeno e hidrogeno & 33 & 22 & 13 & 4 \\
\hline Va para el aire en partículas muy pequeñas & 41 & 55 & 51 & 94 \\
\hline
\end{tabular}

1) Resultados de Osborne y Cosgrove, 1983; 2) Resultados de Zamorano et al., 1994; 3) Resultados en este estudio. 
Sobre el origen de la humedad que empaña la superficie externa de un vaso vedado con hielo (pregunta 3 ), solo el $22 \%$ de los alumnos en este estudio admiten la opción de respuesta que considera la condensación, pero en esto no se muestran diferentes, o hasta un poco mejores, que los de más edad en los estudios anteriores referidos. Sin embargo, el $34 \%$ admite que el agua atraviesa el vidrio, mientras a los de más edad no les parece plausible esta idea.

Con dimensión semejante a la respuesta correcta, el $16 \%$ consideran que el frio hace que el oxígeno y el hidrógeno del aire se transformen en agua, mientras en los estudios anteriores, el porcentaje se eleva a cerca de 50\%. El 19\% considera que "el frio atraviesa el vidrio y se transforma en agua", mientras 34-35\% de los de más edad eligen esta opción. Más de $80 \%$ de los alumnos de más edad, en ambos estudios anteriores referidos, eligen las dos opciones, que presuponen una transformación de naturaleza química. La introducción en este estudio de una opción de respuesta permite constatar que $9 \%$ de los alumnos en los grados 3 ro y 4 tono anticipa la ocurrencia del fenómeno al elegir "El frasco continúa seco por fuera."

Tabla 3 - Resultados en porcentaje obtenidos a cada una de las opciones de respuesta a la pregunta sobre qué pasará con un frasco con hielo con una tapa bien apretada.

\begin{tabular}{|l|c|c|c|c|}
\hline Opciones de respuesta & $\begin{array}{c}1 \\
13 \text { años }\end{array}$ & $\begin{array}{c}2 \\
13 \text { años }\end{array}$ & \multicolumn{2}{|c|}{$\begin{array}{c}\text { 3 } \\
\text { Grados 3 y 4 } \\
\text { Pre - Post }\end{array}$} \\
\hline El frasco continúa seco por fuera & & & 9 & 1 \\
\hline $\begin{array}{l}\text { El agua del hielo que se derrite y pasa a través } \\
\text { del vidrio para fuera }\end{array}$ & 2 & 2 & 34 & 7 \\
\hline $\begin{array}{l}\text { El frio hace que el oxígeno y el hidrogeno del aire } \\
\text { se transformen en agua }\end{array}$ & 50 & 47 & 16 & 2 \\
\hline El agua existente en el aire se pega al vidrio frio & 14 & 16 & 22 & 79 \\
\hline El frio atraviesa el vidrio y se transforma en agua & 34 & 35 & 19 & 10 \\
\hline
\end{tabular}

1) Resultados de Osborne y Cosgrove, 1983; 2) Resultados de Zamorano et al., 1994; 3) Resultados en este estudio.

Las columnas derechas (grados 3 y 4, post test) en las tablas 1,2 y 3 muestran los resultados obtenidos en el post teste, cerca de 5-6 meses después de la realización de la secuencia de actividades. La aplicación de las actividades y la metodología por los profesores participantes con sus alumnos revela un cambio muy destacado de las respuestas de los alumnos en dirección a la opción correcta. Si a la pregunta 1 (tabla 1, columna derecha) no se verifica ningún cambio, además no se le dirigía cualquier actividad específica, en relación con las preguntas 2 y 3 , los cambios son evidentes y estadísticamente significativos en dirección a la opción correcta por la aplicación de T test (opción 2d, p<0,004 y opción $3 d, p<0,0001$ ). En la pregunta 3, aunque el $20 \%$ de los alumnos persista en elegir respuestas que corresponden a ideas alternativas, el $79 \%$ pasa a identificar la condensación. En particular, los alumnos que en pre test consideran que "el frio atraviesa el vidrio y se transforma en agua" revelan un cambio muy limitado estadísticamente no significativo. En su conjunto las respuestas a las diferentes opciones de respuesta en pre y pos test revelan no pertenecer a la misma población $\left(\mathrm{Chi}^{2}, \mathrm{p}<0,0001\right)$. 


\section{Conclusiones}

Los resultados en el pre-test revelan algo sorprendentemente que los alumnos de 3 ro y 4to grados en este estudio tienen claro que las burbujas en el agua hirviente están formadas por vapor, resultado que el post teste confirma. Los alumnos más viejos, en los estudios de Osborne y Cosgrove (1983) y Zamorano et al. (1994), reparten sus respuestas por la totalidad de las opciones.

También se destaca el hecho de que los alumnos en los 3 estudios revelan un mismo perfil en relación a la conservación de la materia. Cerca de la mitad (41-55\%) considera el agua "va para el aire en partículas muy pequeñas". En la investigación del proyecto SPACE (Russel y Watt, 1990) la respuesta a la pregunta abierta ¿para dónde va el agua?, las referencias dominantes son "nubes" y "cielo", pero adicionadas a "aire" suman el 66\%, aunque la muestra sea muy pequeña. Se destaca que los alumnos de 3 ro y 4 to grados en este estudio admiten la conservación del agua en la misma dimensión. La no conservación explicita se evidencia por el hecho que $23 \%$ de los alumnos de más edad continúa a considerar que el agua "simplemente se seca y deja de existir" con valores semejantes a los obtenidos con alumnos de 3 ro y 4 to grados (20\%) en este estudio. El 15\% de los alumnos en este estudio, al considerar que el agua tendrá que ir para algún lado, elijen "Es absorbida por el plato", comparan con los resultados del estudio de Russel y Watt (1990) referido. Comparados los 3 estudios (gráficos 3), es semejante la dimensión de los que perciben la condensación al elegir la opción "El agua existente en el aire se pega al vidrio frio" en la pregunta 3 (tabla 3). En el estudio de Russel y Watt (1990), la condensación no es referida o lo es de modo residual. Los alumnos de 8-9 años, como los de 10-11, se dividen entre considerar que no es posible recuperar el agua evaporada o que se recupera como lluvia. Como ya se hizo referencia, en ausencia de una conceptualización de condensación, surge la precipitación como proceso alternativo (Márquez y Bach, 2007; Gaviria, 2013).

A la pregunta 3, las diferencias entre los tres estudios se encuentran en las opciones alternativas. Algunos autores sugieren la enseñanza escolar y la literatura de divulgación científica sobre la composición del agua, como responsables por la evolución menos positiva de las respuestas de los alumnos de más edad, en que se encuentra como mayoritaria "oxígeno e hidrógeno". La comparación entre los resultados en este estudio y los referidos, apunta en esta dirección. En las 3 preguntas, la opción de respuesta que involucra "oxígeno e hidrógeno", así como la opción que sugiere la transformación del aire en agua por acción del frio, surgen destacadas, agregando más de $80 \%$ de los alumnos con más escolaridad. La escolaridad parece contribuir para el incremento de un entendimiento que al evaporarse el agua se transforma en sus componentes, hidrógeno y oxígeno, como un cambio químico o que el frio promoverá cualquier tipo de transformación que hace surgir agua, como una nueva substancia.

La realización de actividades dirigidas específicamente a las ideas alternativas, buscando que por la investigación y la observación los alumnos refuten y reelaboren sus ideas, ha producido resultados relevantes. Además, se destaca que los profesores aplican las propuestas como resultado de una formación basada no solamente en las actividades "per se", sino en una metodología enfocada en articular las ideas previas de los alumnos como hipótesis de una investigación en que la "teoría corpuscular" del profesor constituye un marco explicativo, que brinda a los alumnos un modelo alternativo en que integran de modo satisfactorio sus observaciones.

El post test muestra una evolución radical en dirección a las opciones deseadas, sin embargo, en la pregunta 3 persisten $10 \%$ que continúan considerando que "El frio atraviesa el vidrio y se transforma en agua". Efectivamente, no se ha propuesto alguna actividad específicamente destinada a refutar esta hipótesis alternativa. Importa además 
destacar que los alumnos sujetos de este estudio revelan en el post test mejores resultados que los de más escolaridad en los dos estudios de referencia. Se puede afirmar que las actividades han logrado el objetivo de proporcionar un mejor nivel de respuesta a cuestiones que exigen una comprensión adecuada de los procesos implicados en los cambios de estado. Los resultados refuerzan la contribución de Johnson (1998 y 2011) y Venville (2004) que sugieren la introducción precoz de la teoría corpuscular de la materia como teoría globalizante sin lo que los alumnos y mismo los adultos, tenderán a elaborar su propia teoría explicativa a partir de su experiencia sensorial y desenvolver teorías robustas basadas en modelos realistas o macroscópicos.

\section{Limitaciones e implicaciones}

Los resultados obtenidos impulsan a adoptar la metodología en la formación de docentes, integrando más actividades para explorar el estado gaseoso y la disolución, bien como introducir elementos complementarios de comunicación multimodal, visual, esquemática y lectura, con refuerzo en la comunicación escrita y oral, en línea con las sugerencias, entre otros, de Johnson y Papageorgiou (2010), buscando una más clara articulación con el ciclo del agua. Aunque este trabajo se integre en un proyecto orientado esencialmente a la producción de propuestas de secuencias de enseñanza, se tendrá, en el contexto de su evaluación, a profundizar en la investigación sobre las representaciones de los alumnos sobre los fenómenos y su relación con el ciclo del agua.

\section{Referencias bibliográficas}

Benarroch, A.B. (2000). El desarrollo cognoscitivo de los estudiantes en el área de la naturaleza corpuscular de la materia. Enseñanza de las Ciencias, 18(2), 235-246

Cachapuz, A., Praia, J. y Jorge, M. (2000). Formação de Professores. Ciências. Perspectivas de Ensino, textos de apoio no 1. Porto: Centro de Estudos de Educação em Ciência (CEEC).

Cachapuz, A., Praia, J. y Jorge, M. (2002). Ciência, Educação em Ciência e Ensino das Ciências - Temas de investigação 26. Lisboa: Ministério da Educação, Portugal.

Cañada Cañada, F., Melo Niño, L. y Álvarez Torres, R. (2013). ¿Qué saben los alumnos de Primaria sobre los sistemas materiales y los cambios químicos y físicos? Campo Abierto, 32(1), 11-33.

Castelltor, A. (2015). Actividades que contribuyen a la promoción de una nueva cultura ambiental del agua. Comunicações, 22(2), 363-389. (ed. Especial 2015) DOI: http:// dx.doi.org/10.15600/2238-121X/comunicacoes.v22n2ep363-389

Dávila Acedo, Ma. A., Cañada Cañada, F. y Sánchez Martín, J.S. (2017). Las ideas previas sobre cambios físicos y químicos de la materia, y las emociones en alumnos de Educación Secundaria. Enseñanza de las Ciencias, Número Extraordinario, 3977-3983.

Forbes, C.T., Zangori, L. y Schwarz, C.V. (2015). Empirical Validation of Integrated Learning Performances for Hydrologic Phenomena: 3rd-Grade Students' Model-Driven Explanation-Construction. Journal of Research in Science Teaching, 52(7), 895-921. DOI: https://doi.org/10.1002/tea.21226

Gaviria, C.M.. (2013). Diseño e implementación de una propuesta de enseñanza y aprendizaje del concepto de materia y sus cambios de estados para estudiantes de quinto grado de básica primaria de la I. E Federico Carrasquilla. Tesis Maestría en Enseñanza de las Ciencias Exactas y Naturales. Universidad Nacional de Colombia, Facultad de Ciencias, Medellín, Colombia. 2013. 
González, Y. L. S. (2015). Enseñanza aprendizaje del concepto ciclo del agua en estudiantes de Básica Primaria. Tese Maestría en Enseñanza de las Ciencias Exactas y Naturales, Universidad Nacional de Colombia (Manizales), Facultad de Ciencias Exactas y Naturales.

Gunckel, K.L., Covitt, B.A., Salinas, I. y Anderson, C.W. (2012). A Learning Progression for Water in Socio-Ecological Systems. Journal of Research in Science Teaching, 49(7), 843-868. https://doi.org/10.1002/tea.21024

Hadenfeldt, J.C., Neumann, K., Sascha Bernholt, S, Liu X. y Ilka Parchmann, I. (2016). Students' Progression in Understanding the Matter Concept . Journal of Research in Science Teaching, 53(5), 683-708. DOI: http://dx.doi.org/10.1002/tea.21312

Johnson, P. (1998). Progression in children's understanding of a 'basic' particle theory: a longitudinal study, International Journal of Science Education, 20(4), 393-412. DOI: http://dx.doi.org/10.1080/0950069980200402

Johnson, P. y Papageorgiou, G. (2010) Rethinking the Introduction of Particle Theory: A Substance-Based Framework. Journal of Research in Science Teaching, 47(2), 130-150. https://doi.org/10.1002/tea.20296

Johnson, P. y Tymms, P. (2011). The Emergence of a Learning Progression in Middle School Chemistry. Journal of Research in Science Teaching, 48(8), 849-877. DOI: http:// dx.doi.org/10.1002/tea.20433

Kind, V. (2004). Más allá de las apariencias. Ideas previas de los estudiantes sobre conceptos básicos de química. Aula XXI. México: Editorial Santillana. ISBN: 970-29-1232-6

Márquez, C y Bach, J (2007). Una propuesta de análisis de las representaciones de los alumnos sobre el ciclo del agua. Enseñanza de las Ciencias de la Tierra, 15(3), 280286.

Nebot, M.R. y Márquez, C. (2017). Uso del modelo cinético-corpuscular en explicaciones sobre fenómenos naturales. Enseñanza de las Ciencias, Número extraordinario, 4287-4292.

Osborne, R. y Cosgrove, M. (1983) Children's conceptions of the changes of state of water. Journal of Research in Science Teaching, 20(9), 825-838. DOI: https://doi. org/10.1002/tea.3660200905

Piedras, S. C. y Mota, A.D.L. (2011). Elementos para el diseño de una estrategia didáctica relacionada con la construcción de un modelo cinético escolar sobre los cambios de estado de la materia. XI Congreso Nacional de Investigación Educativa / 5 . Educación y Conocimientos Disciplinares / Ponencia. 7 a México, DF: Consejo Mexicano de Investigación Educativa, A.C. 11 Noviembre 2011. Recuperado de: http://www. comie.org.mx/congreso/memoriaelectronica/v11/docs/area_05/2488.pdf

Russell, T. y Watt, D. (1990). SPACE Project Research Report. Evaporation and Condensation. Liverpool: Liverpool University Press, 1990.

Stavy, R. (1990). Children's conception of changes in the state of matter: From liquid (or solid) to gas. Journal of Research in Science Teaching, 27(3), 247-266. DOI: https:// doi.org/10.1002/tea.3660270308

Varelas, M.P., Christine, C. y Rife, A. (2006). Exploring the role of intertextuality in concept construction: Urban second graders make sense of evaporation, boiling, and condensation. Journal of Research in Science Teaching, 43(7), 637-666. DOI: https:// doi.org/10.1002/tea.20100 
Venville, G. (2004). Young Children Learning about Living Things: A Case Study of Conceptual Change from Ontological and Social Perspectives. Journal of Research in Science Teaching, 41(5), 449-480. DOI: https://doi.org/10.1002/tea.20011

Zamorano, R., Dell'Oro, G., y Silva, N. (1994). Diagnostico de ideas previas en Física. Revista de Enseñanza de la Física, 7(2), 35-40. Recuperado de: https://revistas.unc.edu.ar/ index.php/revistaEF/article/view/16215/16056 


\section{ANEXO}

Preguntas del cuestionario (columna izquierda) y resultados en el pre teste en cada una de las siete clases involucradas (columna derecha)

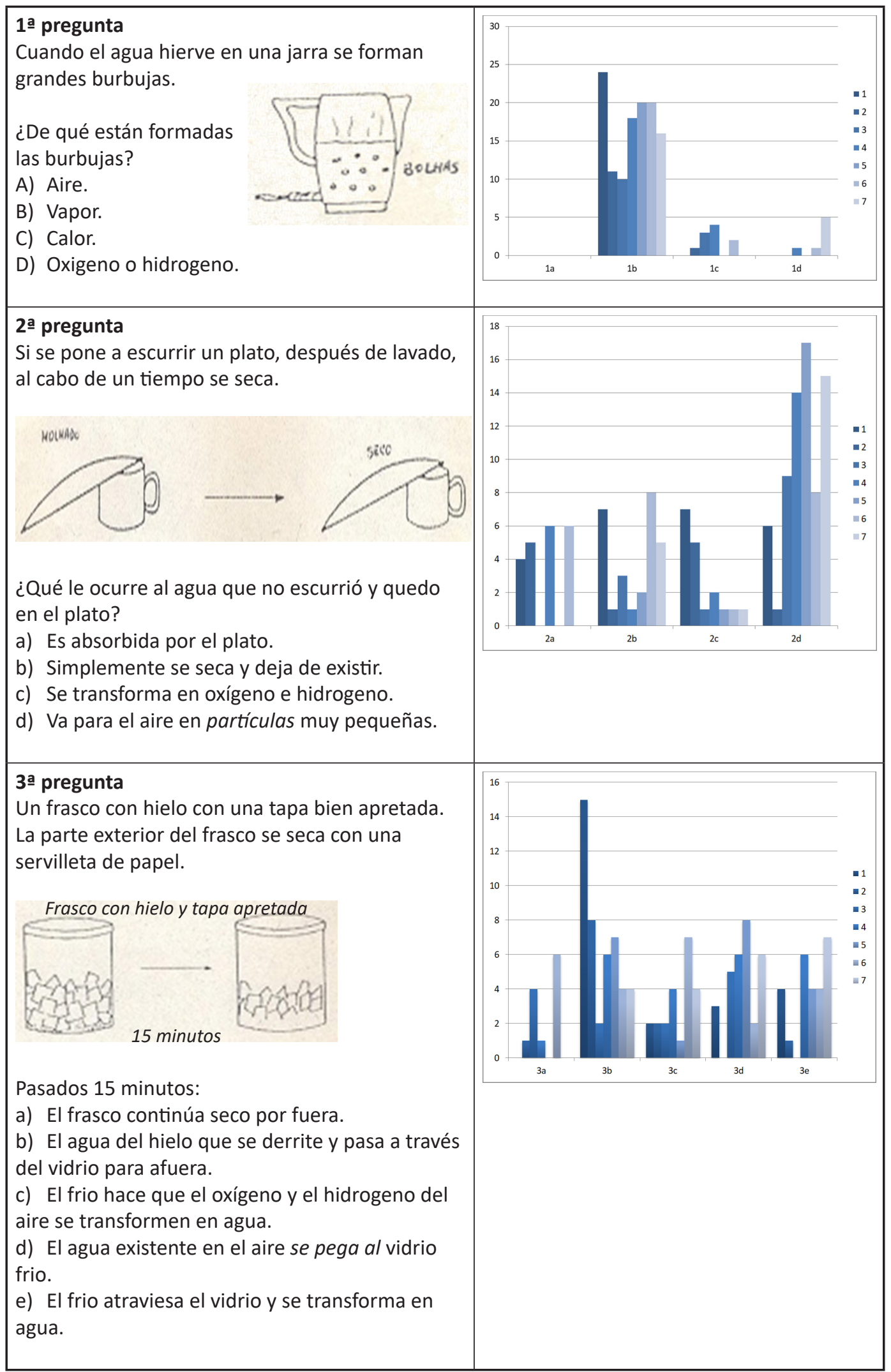


